\title{
Aus der Alten Aktienspinnerei wird die neue Universitätsbibliothek Chemnitz
}

\author{
Angela Malz
}

Universitätsbibliothek Chemnitz, orcid.org/0000-0001-7320-1231

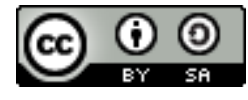

Dieses Werk und die Abbildungen 14 und 16 ist lizenziert unter einer Creative Commons Namensnennung - Weitergabe unter gleichen Bedingungen 4.0 International Lizenz. (https://creativecommons.org/licenses/by-sa/4.0/) Alle anderen Abbildungen sind von der Lizenz ausgenommen. Die Nutzung ist beim Rechteinhaber zu erfragen.

\section{Geschichte der Alten Aktienspinnerei}

Chemnitz im Jahr 1857 - am Rande der Stadt wird am 19.05. des Jahres der Grundstein für eine Spinnerei gelegt. Chemnitz ist zu dem Zeitpunkt eine aufstrebende Industriestadt. Vor allem Betriebe des Maschinenbaues und der Textilindustrie prägen das Stadtbild. Einer der bis heute bekannten Industriellen, Richard Hartmann, hatte 1852 die Idee für den Bau dieser Spinnerei. Der Kaufmann August Götze und die Bankiers Carl Knackfuß und Moritz Ferdinand Bahse setzten die Idee in die Tat um und kauften ein 10 ha großes Grundstück, auf dem die Spinnerei gebaut wurde. Am 30.3.1857 wurde die Chemnitzer-Actien-Spinnerei gegründet und nach nur zweijähriger Bauzeit war das Gebäude fertig. Entstanden war die größte Spinnerei Sachsens mit 1100 Arbeitsplätzen und 60000 Spindeln. Die Organisation der Spinnerei als Aktiengesellschaft war der Grund dafür, dass das Gebäude anfangs als „Aktienspinnerei“ und später als „Alte Aktienspinnerei“ bezeichnet wurde.

Die Alte Aktienspinnerei wurde im Baustil des historischen Eklektizismus errichtet. Der Architekt Friedrich Theodor Roschig hatte das Gebäude aufgrund der Brandgefahr ganz aus Eisen und Stein projektiert und auf Holz als Baumaterial verzichtet. Das Gebäude zählte damals zu den brandsichersten der Stadt Chemnitz. Über ein Viadukt war das Gebäude mit dem Bahnhof Chemnitz verbunden. 


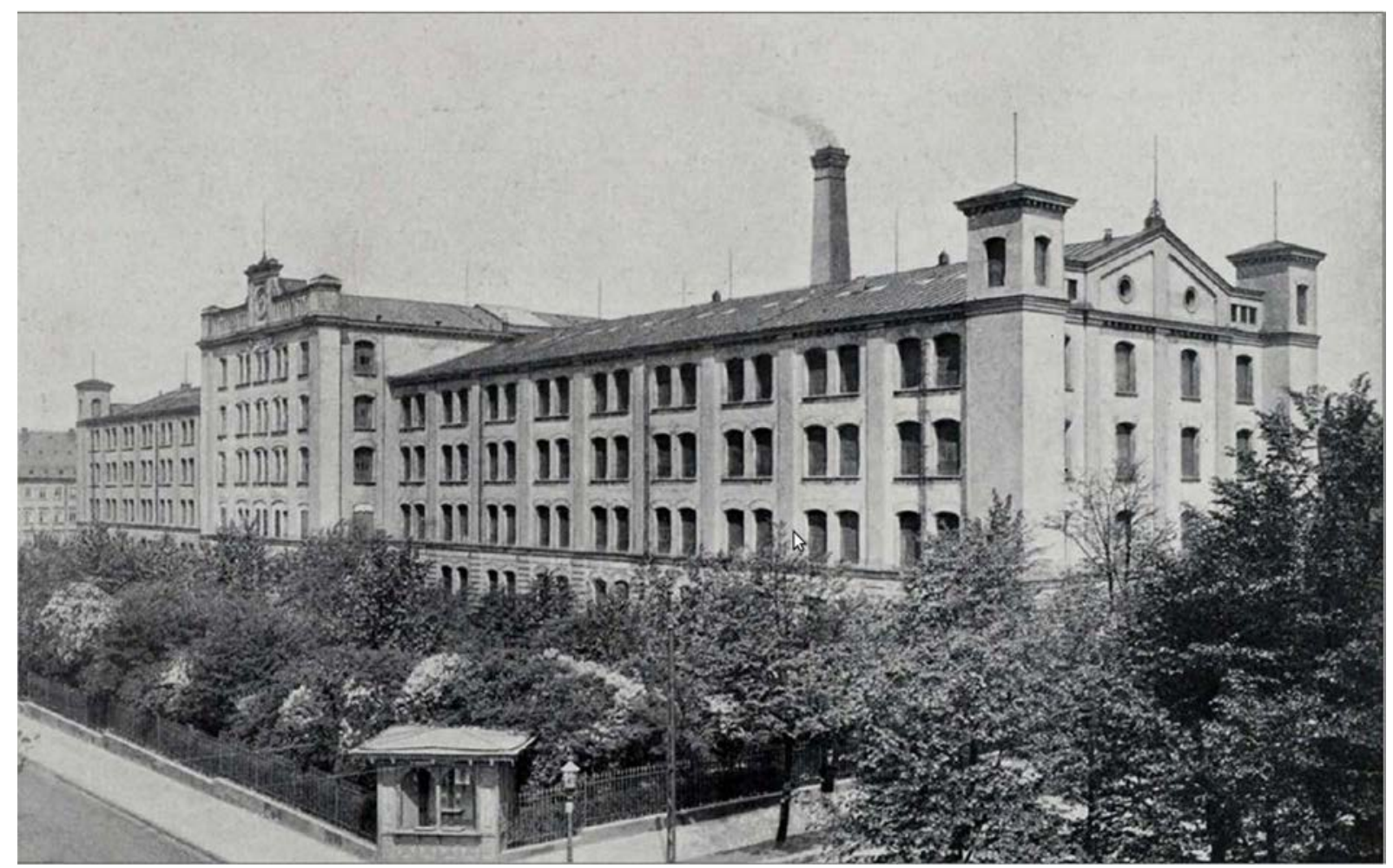

Bild 1: Historische Aufnahme der Alten Aktienspinnerei, Quelle: Stadtarchiv Chemnitz

Die Produktion wurde 1860 aufgenommen mit 972000 Pfund Garn. Schnell stieg die Produktivität und 1867 produzierte man schon 1671000 Pfund Garn bei unveränderter Spindelzahl von 60000 . In den Jahren 1887 bis 1891 ersetzte man die alten Spinnmaschinen durch neue aus englischer Produktion. Durch die gestiegene Produktionsmenge wurde die Alte Aktienspinnerei zu klein und schrittweise zog die Spinnerei in ein anderes Gebäude in Altchemnitz. Bis 1914 war die gesamte Produktion verlagert und am 4.7.2029 wurde die Aktiengesellschaft schließlich aufgelöst.

Nach dem Auszug der Spinnerei wurde das Gebäude vielfach genutzt. Recherchen in den einschlägigen Archiven ergaben mehr als 80 Nutzer. Dazu gehörten Betriebe der Textilindustrie aber auch ein Lager für Zwangsarbeiter in den Jahren 1943 bis 1944.

Im 2. Weltkrieg wurde das Haus stark beschädigt und verlor die oberste Etage. Nach dem Krieg bekam das Gebäude ein Notdach und erlebte eine vielfältige Nutzung: u.a. als Wismut Kaufhaus Glückauf (1950 bis 1990) und EDEKA-Aktiv-Markt (1991 bis 1996), als Städtisches Puppentheater (1955 bis 1996), als Domizil des VEB Kraftverkehr (von 1962 bis 1998), als Stadtbibliothek (von 1950 bis 2004) und nach der Jahrtausendwende auch als Galerie.

Mit dem Auszug des letzten Nutzers - der Neuen Sächsischen Galerie - stand das Haus ab 2004 leer und verfiel zusehends. 


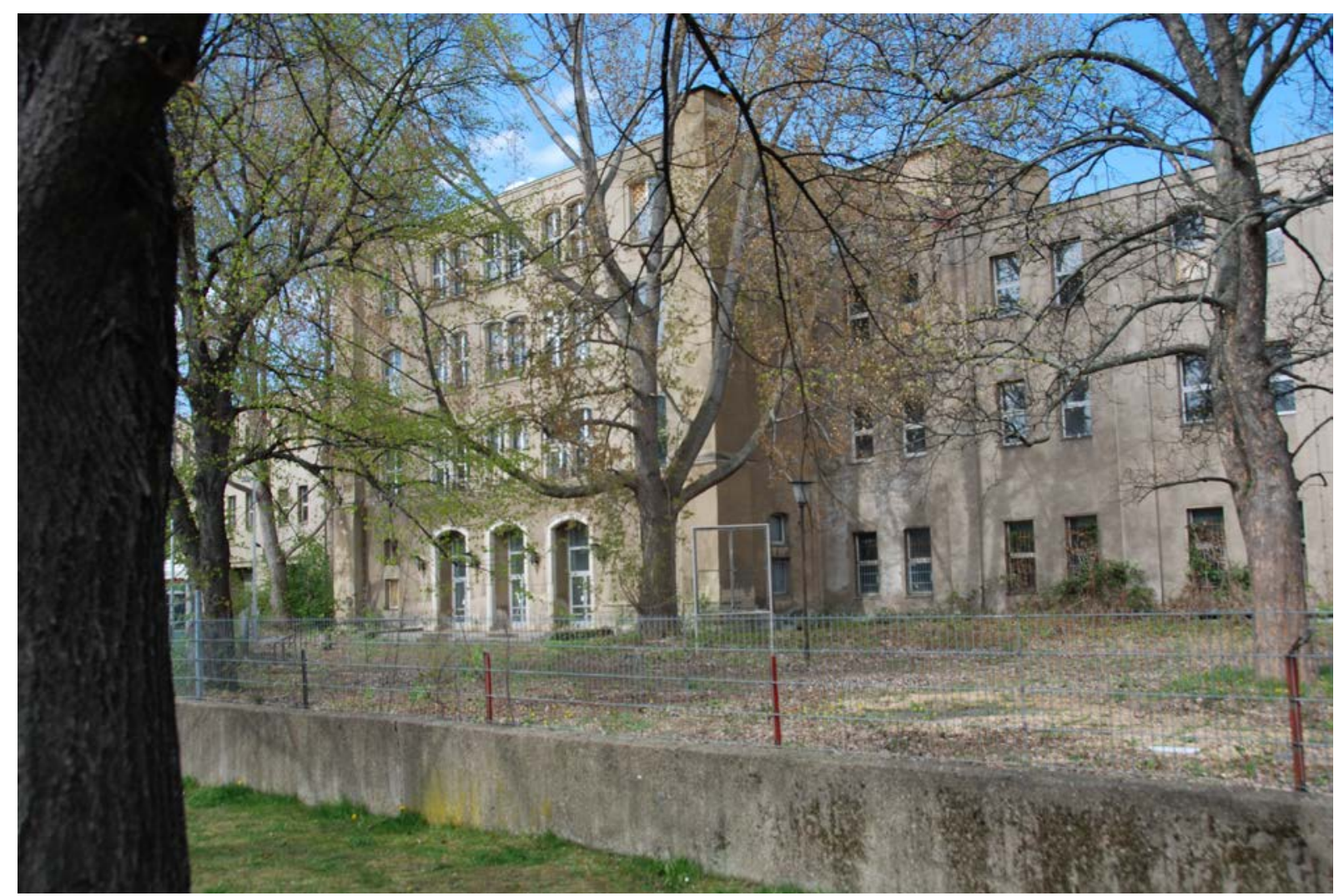

Bild 2: Außenansicht der Alten Aktienspinnerei 2014, Quelle: TU Chemnitz / M. Steinebach

\section{Chemnitz als Stadt der Wissenschaft}

Im Jahr 2009 entstand die Idee zu einer Bewerbung der Stadt Chemnitz als „Stadt der Wissenschaft 2011“. Während dieser Zeit wurde viel darüber nachgedacht, wie Wissenschaft und die Stadt Chemnitz noch besser verzahnt werden können. Innerhalb der Aktion „Denken im Zentrum“ entstand die Idee, die Alte Aktienspinnerei für die Universitätsbibliothek nutzbar zu machen. Unterstützt wurde dieser Gedanke im Juni 2010 durch ein städtebauliches Entwicklungskonzept.

Mit der Idee, die Universitätsbibliothek in der Alten Aktienspinnerei unterzubringen, war auch der Plan verbunden, studentisches Leben in die Innenstadt zu bringen. Die Technische Universität Chemnitz ist über mehrere Standorte über das gesamte Stadtgebiet verteilt, jedoch fehlt der Innenstadt das Flair einer Universitätsstadt. Außerdem soll der an die Aktienspinnerei angrenzende Stadtteil „Brühl“ wieder zum Leben erweckt und studentisches Leben in generationsübergreifendes Wohnen integriert werden. In den Planungen stellte man den Anspruch an die Universitätsbibliothek, zum Motor der Stadtentwicklung zu werden.

Die Technische Universität Chemnitz forcierte die Idee, die Universitätsbibliothek in der Aktienspinnerei unterzubringen und stellte im September 2010 eine Bedarfsanmeldung beim zuständigen Ministerium. Schon im Oktober 2010 erhielt die TU Chemnitz die bestätigte Bedarfsanmeldung für eine Bibliothek mit 12575 m² Hauptnutzfläche.

Chemnitz hat den Titel "Stadt der Wissenschaft 2011“ nicht bekommen. Aber die Idee, die Aktienspinnerei in die Universitätsbibliothek zu verwandeln, hat überlebt. Ein wichtiger Schritt zur Verwirklichung des Planes wurde 2011 gemacht. Der Freistaat Sachsen erwarb das Gebäude und damit war der Weg frei, die Alte Aktienspinnerei zur Universitätsbibliothek umzubauen. 


\section{Die TU Chemnitz}

Die TU Chemnitz hat aktuell knapp 10400 Studierende und 1150 Personalstellen. In acht Fakultäten (Naturwissenschaften, Mathematik, Maschinenbau, Elektrotechnik und Informationstechnik, Informatik, Wirtschaftswissenschaften, Human- und Sozialwissenschaften sowie in der Philosophischen Fakultät) werden ca. 100 Studiengänge angeboten. Diese Studiengänge zeichnen sich durch ein hohes Maß an Interdisziplinarität aus. Die TU Chemnitz ist eine sehr internationale Universität und hat den höchsten Anteil an ausländischen Studierenden aller sächsischen Hochschulen und Universitäten.

Für ihre Forschung und Lehre hat die TU Chemnitz drei Kernkompetenzen definiert: „Materialien und Intelligente Systeme“, „Ressourceneffiziente Produktion und Leichtbau“ sowie "Mensch und Technik“.

\section{Die Universitätsbibliothek Chemnitz und ihre bauliche Situation}

Die UB Chemnitz versteht sich als digitale Bibliothek, die einen Großteil ihres Etats in den Erwerb elektronischer Medien investiert. Seit mehr als 20 Jahren engagiert sich die Bibliothek in der OpenAccess-Bewegung, hält einen Volltextserver vor, eine Universitätsbibliographie, beteiligt sich an den DEAL-Verträgen und unterhält einen Publikationsfonds. Sehr früh entwickelte die UB Aktivitäten zur Vermittlung von Informationskompetenz und zu E-Learning-Angeboten. Sie betreibt den einzigen Universitätsverlag in Sachsen. Die Bibliothek ist innerhalb der Universität, regional und national gut vernetzt und war jahrelang Leadpartner in sächsisch-tschechischen Bibliotheksprojekten. Nur ein zeitgemäßes Bibliotheksgebäude fehlt.

Die Universitätsbibliothek Chemnitz ist dezentral an drei Standorten untergebracht, wobei keines der Gebäude als Bibliotheksgebäude gebaut worden war. Das Konzept „Bibliothek als Lern- und Kommunikationsort" kann nur sehr punktuell umgesetzt werden, weil dafür die räumlichen Voraussetzungen fehlen. Auch die Stellflächen für die gedruckten Bestände sind völlig ausgereizt, obwohl der Zugang an gedruckten Büchern in den letzten Jahren rapide zurückgegangen ist und der Fokus des Bestandsmanagements auf dem Ausbau der Digitalen Bibliothek liegt. An einer Änderung der Situation wurde schon sehr lange gearbeitet. Es gab viele Pläne für einen Bibliotheksneubau oder -umbau, die letztlich aber alle scheiterten.

Das Universitätsarchiv - seit 2014 eine Abteilung der UB - platzt seit langem aus allen Nähten und nutzt verschiedene Flächen der TU Chemnitz an drei verschiedenen Universitätsstandorten.

Der Bedarf nach einem neuen Gebäude ist groß und die Zentralisation aller Bibliotheksstandorte inklusive Universitätsarchiv ein lang gehegter Wunsch.

\section{Vorbereitungen zum Umbau}

Am 2. Mai 2011 beging die TU Chemnitz ihr 175jähriges Jubiläum. In seine Rede anlässlich der Festveranstaltung signalisierte der damalige sächsische Ministerpräsident Stanislaw Tillich den Umbau der Alten Aktienspinnerei zur zentralen Universitätsbibliothek. Das war ein starkes Zeichen und die Idee nahm weiter Gestalt an.

Die Universitätsbibliothek war vorbereitet. Im Jahr 2010 hatte eine Nutzerbefragung stattgefunden und es gab sehr viele Anregungen zur Verbesserung der räumlichen Bedingungen. Außerdem hatten im Frühjahr 2011 die UB-Mitarbeitenden unter dem Thema „Wie stellen Sie sich Ihre 
Wunschbibliothek vor?“ jede Menge Ideen für eine neue Bibliothek geliefert. Im Mai 2011 bildete sich eine Arbeitsgruppe in Vorbereitung des Umbaus. In ihr arbeiteten Vertreter und Vertreterinnen aus der Universitätsleitung, der Universitätsbibliothek, dem Universitätsarchiv, dem Universitätsrechenzentrum, dem Studierendenrat und dem Dezernat Technik zusammen. Diese Vorbereitungen zahlten sich enorm aus, denn am 20.7.2011 wurde der Planungsauftrag an die TU Chemnitz erteilt und im Juli und August wurde die Nutzerforderung erarbeitet. Ohne die vielen Anregungen aus den oben genannten Aktionen wäre es nicht möglich gewesen, innerhalb dieser kurzen Zeit eine strategisch durchdachte Nutzerforderung zu formulieren. Diese wurde am 8.9.2011 dem Sächsischen Ministerium für Wissenschaft und Kunst übergeben.

Ende 2011 kam es zu einer Erweiterung der Nutzerforderung. Für das Erdgeschoss der zu planenden Bibliothek wünschte die Universitätsleitung eine bibliotheksfremde Nutzung, um die Universitätsbibliothek noch besser mit der Universität zu verzahnen. Gedacht wurde u.a. an die Unterbringung des Studentenservices und des Welcome Centers.

Im Jahr 2012 wurde ein europaweiter Wettbewerb zum Umbau der Alten Aktienspinnerei gestartet. In der Ausschreibung gab es die klare Vorgabe, dem Haus seine alte Kubatur wiederzugeben und den Stil eines Industriegebäudes zu belassen.

Aus den 117 Bewerbern aus mehreren europäischen Ländern, die sich für diese Planungsaufgabe beworben hatten, wurden 30 Büros zur Teilnahme ausgewählt. Davon reichten 26 ihren Entwurf für den Wettbewerb ein.

Am 31. Januar 2013 fällte die Jury unter dem Vorsitzenden Professor Paul Kahlfeldt die Entscheidung, den Entwurf der Bietergemeinschaft Lungwitz, Heine, Mildner (Dresden) und Rabe (Berlin) auf Platz 1 zu setzen.

Die Bietergemeinschaft, die sich fortan ARGE Aktienspinnerei nannte, übernahm in Zusammenarbeit mit dem Bauherrn, dem Sächsischen Immobilien- und Baumanagement (SIB) und der TU Chemnitz die weiteren Planungsarbeiten. Im Februar 2014 war wie gefordert die Entscheidungsgrundlage in der Qualität der Entwurfsunterlage Bau fertig. Allerdings musste sie optimiert werden, weil bedingt durch den Plan, das Erdgeschoss bibliotheksfremd zu nutzen, die gewährte Hauptnutzfläche und damit die Baukosten überschritten waren. Deshalb wurde die bibliotheksfremde Nutzung gestrichen und die Hauptnutzfläche wieder auf $12575 \mathrm{~m}^{2}$ reduziert. Die ARGE Aktienspinnerei legte 22 Entwurfsvarianten vor, bis die neue Entwurfsunterlage EW Bau im Juli 2014 fertig war. Diese wurde am 3.12.2014 genehmigt und der Weg zum Umbau der Alten Aktienspinnerei war frei.

\section{Abbruch}

Zu Beginn des Jahres 2015 wurde die Ausführungsunterlage Bau erarbeitet und der Baubeginn auf Juni 2015 festgelegt. Allerdings durften notwendige Abbrüche schon vorher erfolgen.

Die verschiedenen Nutzer hatten im Gebäude Spuren hinterlassen. Es gab jede Menge Anbauten und im Inneren waren unzählige Wände eingezogen wurden. Ab April 2014 wurden leerstehende Gebäude rund um das Hauptgebäude entfernt. Im Oktober 2014 begann die Beräumung der Seitenflügel und alle Einbauten inklusive Fußbodenaufbau wurden in den Rohzustand versetzt. Das gusseiserne Tragwerk mit dem gemaierten Kappengewölbe und die Außenwände wurden konserviert. 
Untersuchungen der Bausubstanz ergaben, dass der Mittelbau komplett entkernt werden musste. Die Außenwände, die erhalten werden konnten, wurden während der Entkernung statisch abgesichert und im Anschluss die Baugrube für den Mittelbau und den Magazinanbau hergestellt.

Auch die Giebelseiten mussten abgebrochen werden.

Insgesamt wurden mehr als 6.500 Tonnen Bauschutt meist in Handarbeit aus dem Gebäude gebracht.

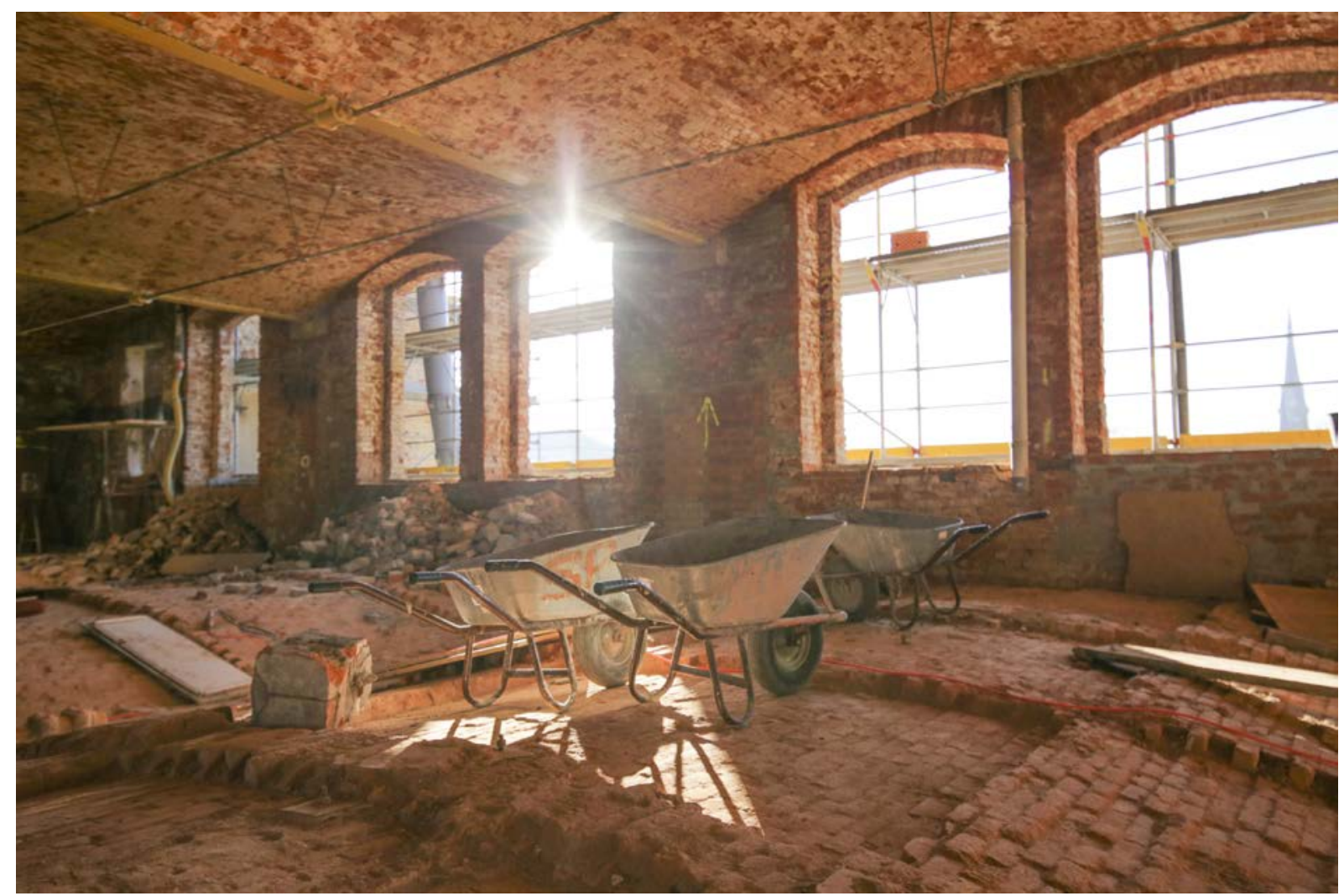

Bild 3 Entkernung der Seitenflügel in Handarbeit, Quelle: TU Chemnitz / J. Müller 


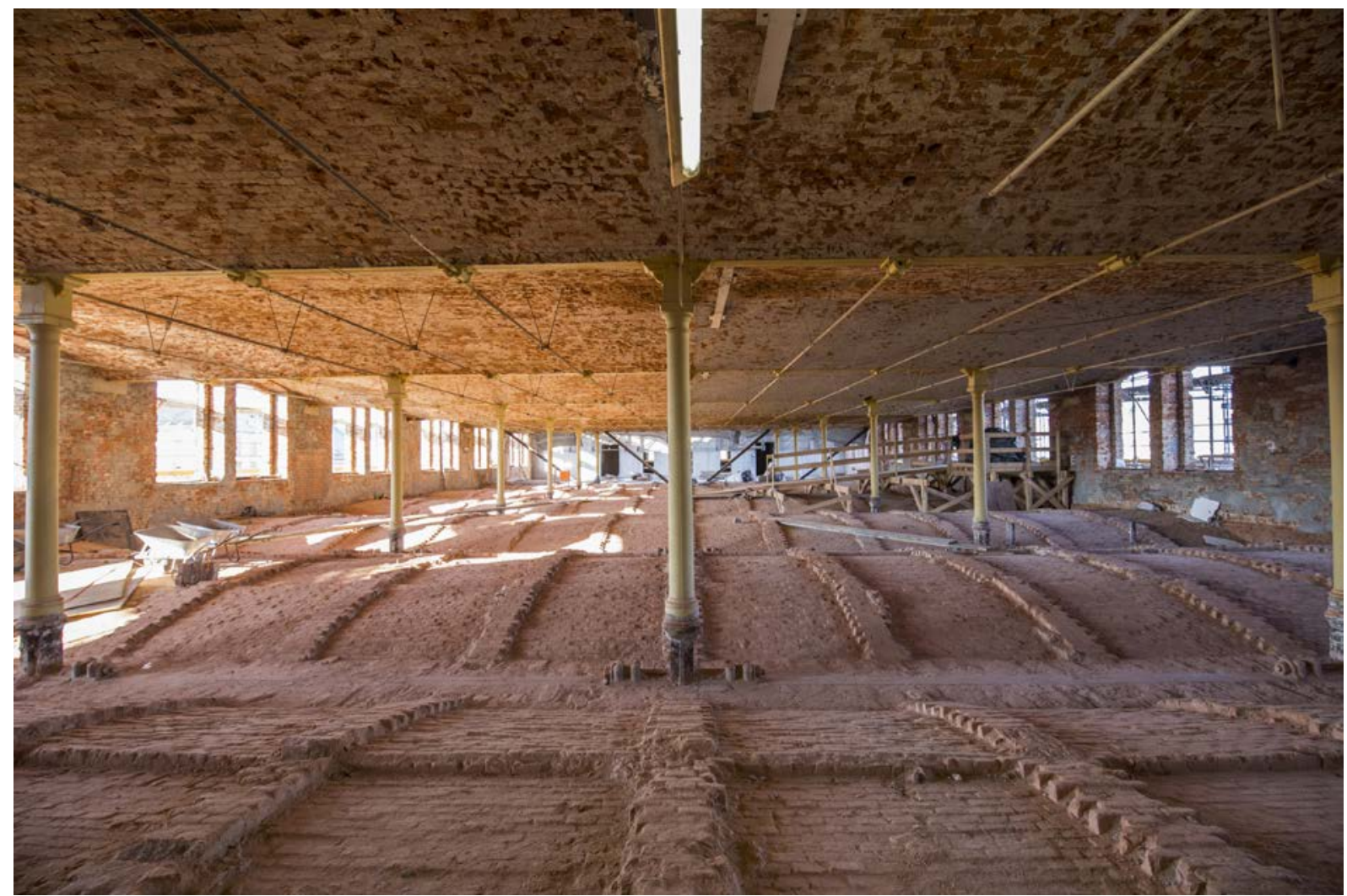

Bild 4 Entkernter Seitenflügel. Gut sichtbar sind die gusseisernen Säulen und die Gewölbe

Quelle: TU Chemnitz / J. Müller

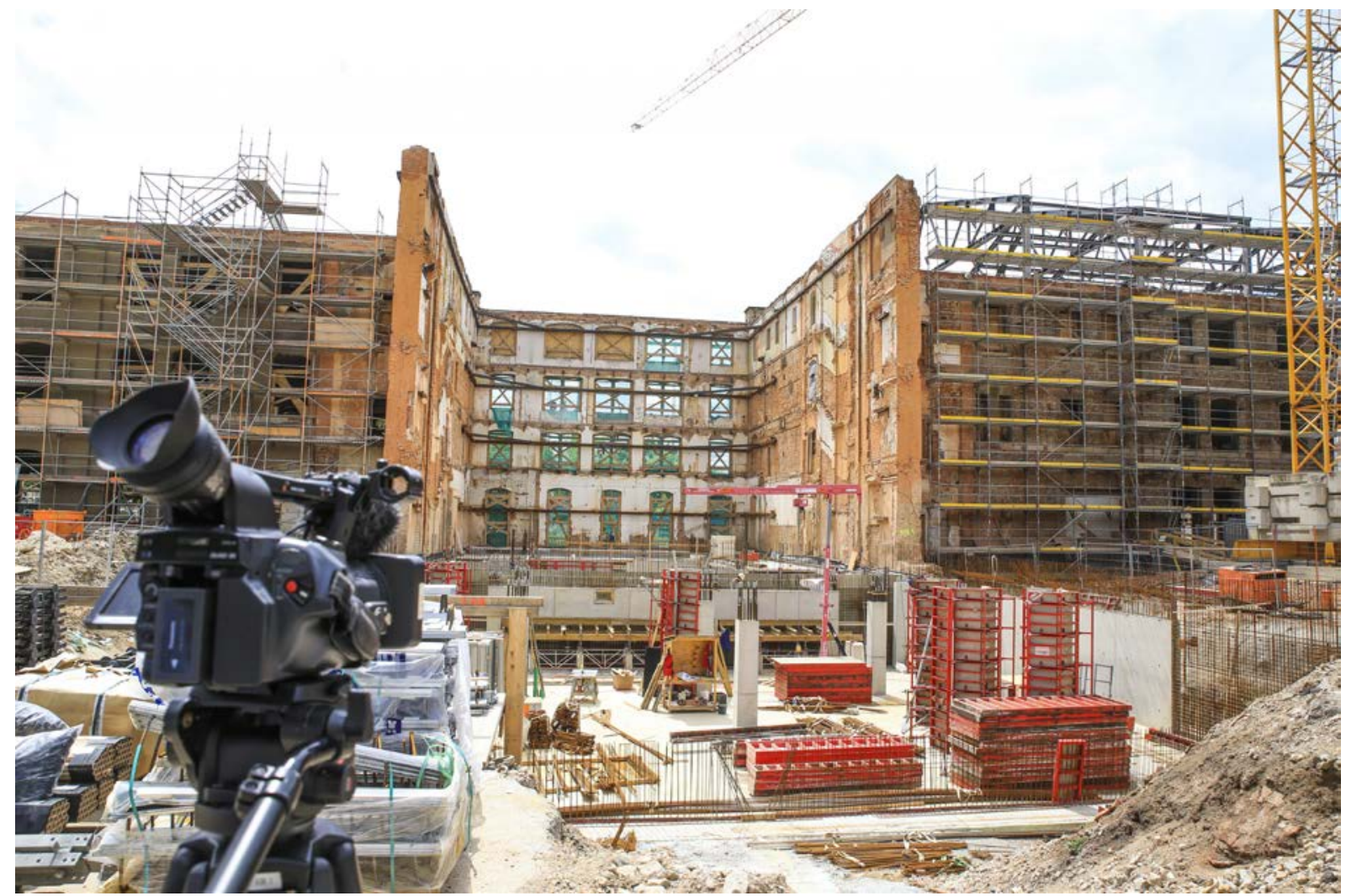

Bild 5 Entkernter Mittelbau, Quelle: TU Chemnitz / J. Müller 


\section{Aufbau}

Im Sommer 2015 konnte mit dem Wiederaufbau und dem Ausbau der erhaltenen Gebäudeteile begonnen werden.

Die Ziele des Umbaus der Alten Aktienspinnerei waren, einerseits eine moderne Bibliothek zu errichte, andererseits aber den Charakter des Gebäudes als Industriebau mit seinen markanten Eigenschaften zu erhalten.

Deshalb musste das Gebäude an die neue Nutzung als Bibliothek angepasst werden.

Die Kubatur des Gebäudes sollte in der ursprünglichen Form wieder hergestellt werden. Diese besteht aus einem zentralen fünfgeschossigen Mittelbau sowie zwei langgestreckten, viergeschossigen Seitenflügeln mit Satteldach und markanten Ecktürmen.

In den Seitenflügeln konnte die denkmalpflegerisch wertvolle Tragstruktur erhalten bleiben. Allerdings musste sie statisch verstärkt und ertüchtigt werden. Der Mittelbau und die äußeren Giebelfelder wurden entkernt und wieder aufgebaut, so wie es die Nutzung als Bibliothek erfordert.

An den Mittelbau wurde der Magazinanbau gesetzt, weil Magazinräume aufgrund der fehlenden Fläche im ursprünglichen Gebäude nicht unterzubringen waren.

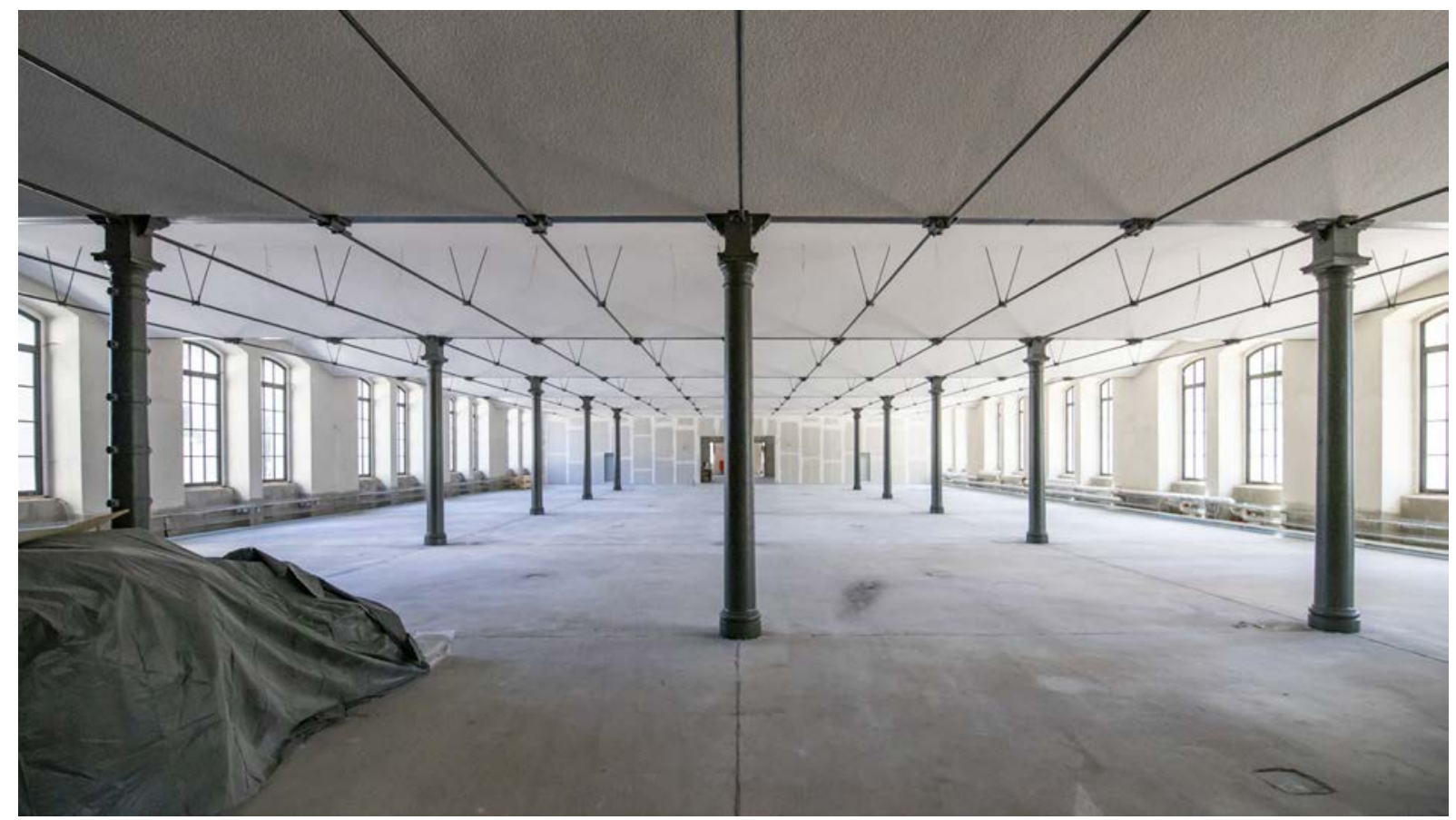

Bild 6 Seitenflügel im Rohbau, Quelle: TU Chemnitz / J. Müller 


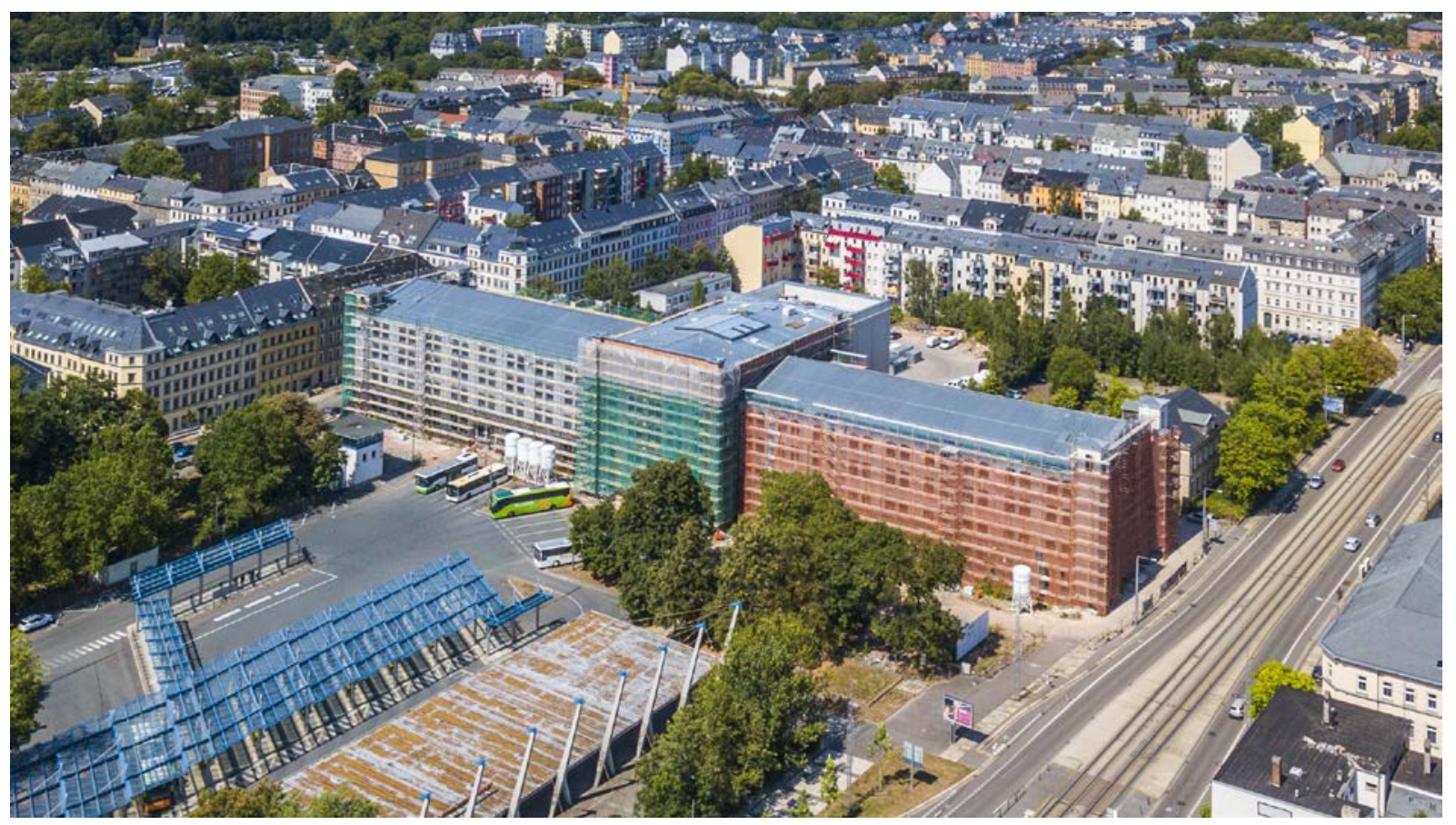

Bild 7 Aktienspinnerei von oben im Rohbau, Quelle: TU Chemnitz / J. Müller

\section{Die Struktur der Bibliothek in der Alten Aktienspinnerei}

Bedingt durch die Gebäudestruktur ist die Bibliothek sehr symmetrisch aufgebaut.

Im Mittelteil, der auch als Herz der Bibliothek bezeichnet wird, befinden sich in der Reihenfolge der Geschosse - beginnend mit dem Untergeschoss - die Garderobe, ein großzügiges Foyer, der Ausleihund Auskunftsbereich, ein Lesesaal mit Empore und im 4. Obergeschoss 20 Carrels.

In den an den Mittelteil angrenzenden Seitenflügeln befinden sich die Freihandbereiche mit integrierten Leseplätzen. An den Giebelseiten sind die Büros untergebracht.

Die Geschosse im Detail:

Das Untergeschoss besteht nur aus dem Mittelteil, weil die Seitenflügel nicht unterkellert sind. Untergebracht sind hier die Garderobe und Technikbereiche.

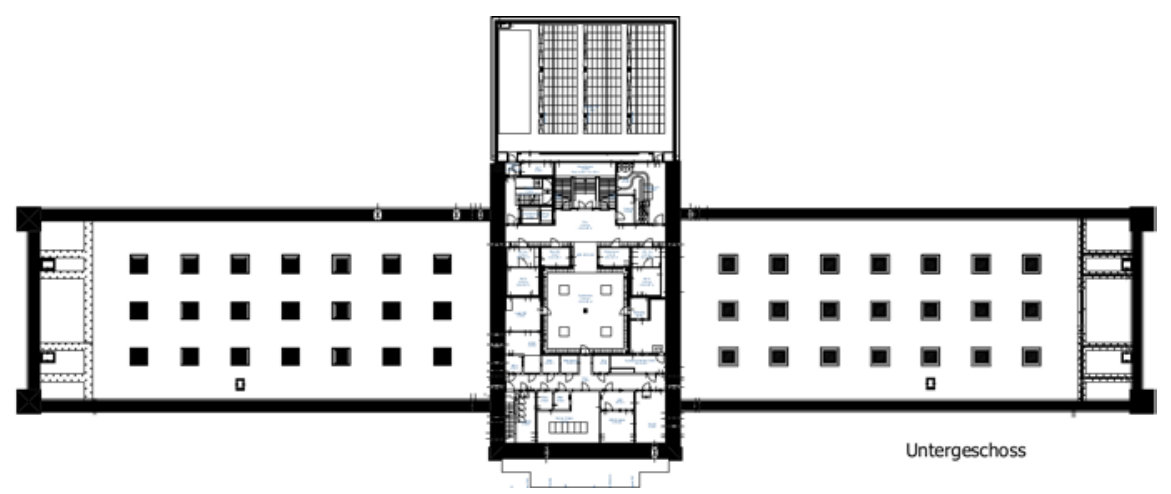

Bild 8

Grundriss des Untergeschosses, Quelle: ARGE Aktienspinnerei 
Im Erdgeschoss befindet sich neben dem Haupteingang eine Wachkanzel, die auch für die Gebäudeüberwachung genutzt wird. Im Foyer ist ein Informationstresen für die Erstauskunft untergebracht. Ein Seitenflügel wird als Freihandbereich mit integrierten 4-Personen-Arbeitstischen genutzt. Der andere Seitenflügel wird ein Lern- und Kommunikationsbereich sein. Angrenzend gibt es vier Gruppenarbeitsräume, die mit variablen Wänden ausgestattet sind. Diese Räume können entweder zu zwei Räumen oder zu einem Raum zusammengeschaltet werden und als Veranstaltungsfläche genutzt werden. Wenn Veranstaltungen stattfinden, wird ein Veranstaltungseingang geöffnet, der in ein kleines Foyer führt.

Im Übergang zum Magazinanbau befinden sich zwei Rückgabeautomaten, die an eine Buchtransportanlage angeschlossen sind. Dieser Teil der Bibliothek ist über einen separaten Ein- und Ausgang begehbar, der auch für den Zutritt zum Lesegarten genutzt werden kann.

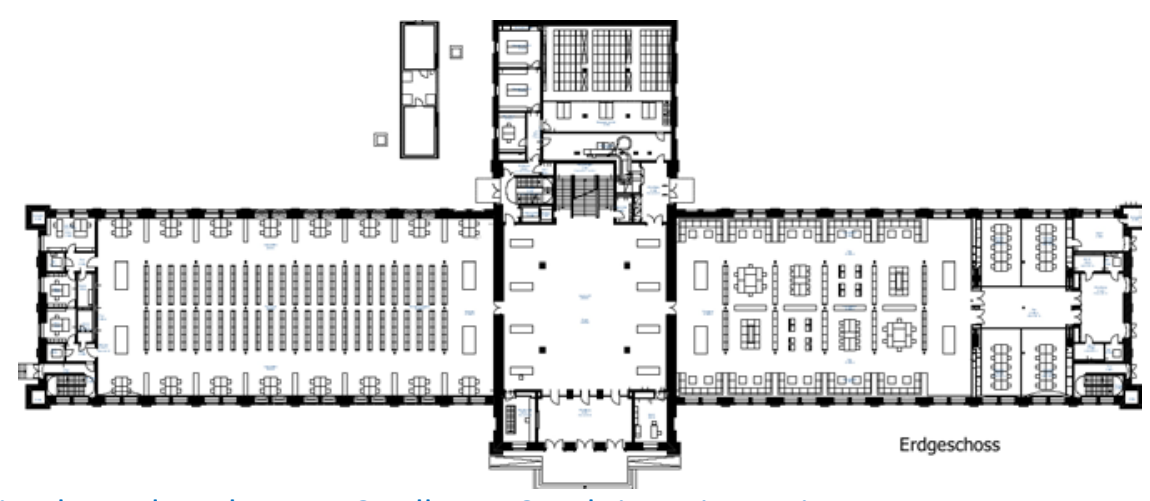

Bild 9 Grundriss des Erdgeschosses, Quelle: ARGE Aktienspinnerei

Im 1. Obergeschoss befindet sich im Mittelteil der Thekenbereich für Ausleihe und Auskunft und die Selbstverbuchungsautomaten. In diesem Bereich sollen auch Vitrinen für Ausstellungen aufgestellt und ein Galeriesystem für Bilder angebracht werden. Angrenzend an diesen Bereich wurde ein Schulungsraum eingerichtet. In den Seitenflügeln befinden sich die Freihandbereiche mit Einzelarbeitsplätzen.

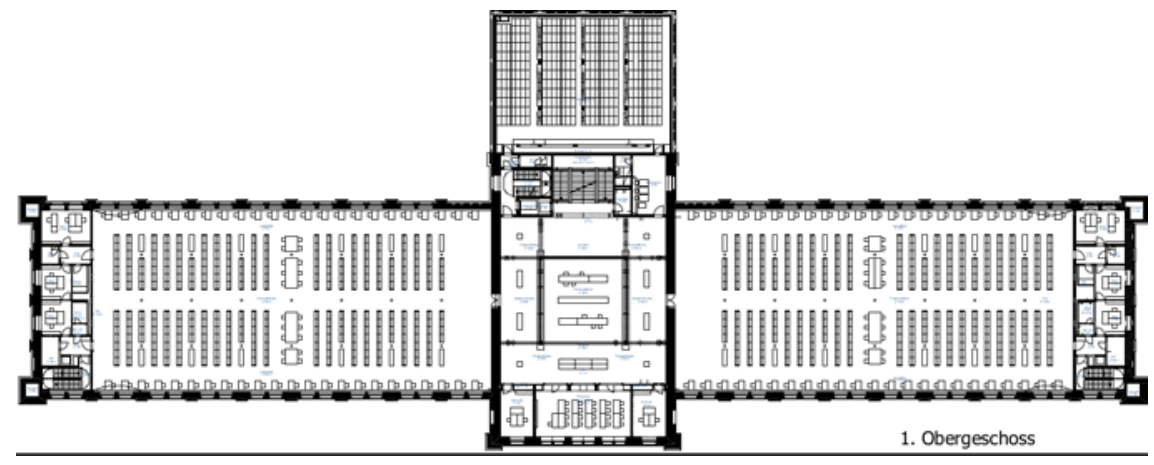


Im 2. Obergeschoss ist der Lesesaal verortet, angrenzend ein zweiter Schulungsraum. Auch in diesem Geschoss befinden sich in den Seitenflügeln Freihandbereiche mit Einzelarbeitsplätzen.

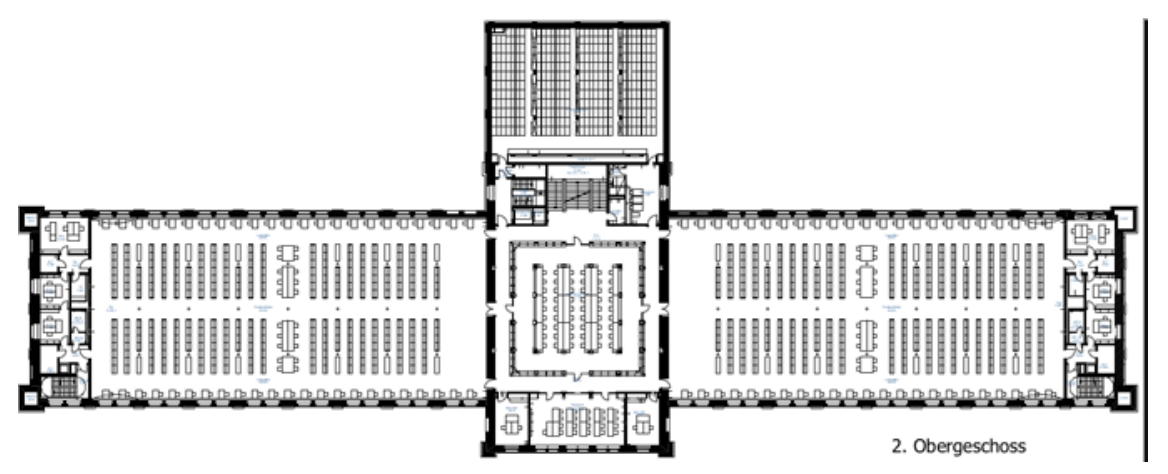

Bild 11 Grundriss des 2. Obergeschosses, Quelle: ARGE Aktienspinnerei

Im 3. Obergeschoss befindet sich die Empore des Lesesaales, ein Beratungsraum und wieder in den Seitenflügeln Freihandbereiche mit Einzelarbeitsplätzen.

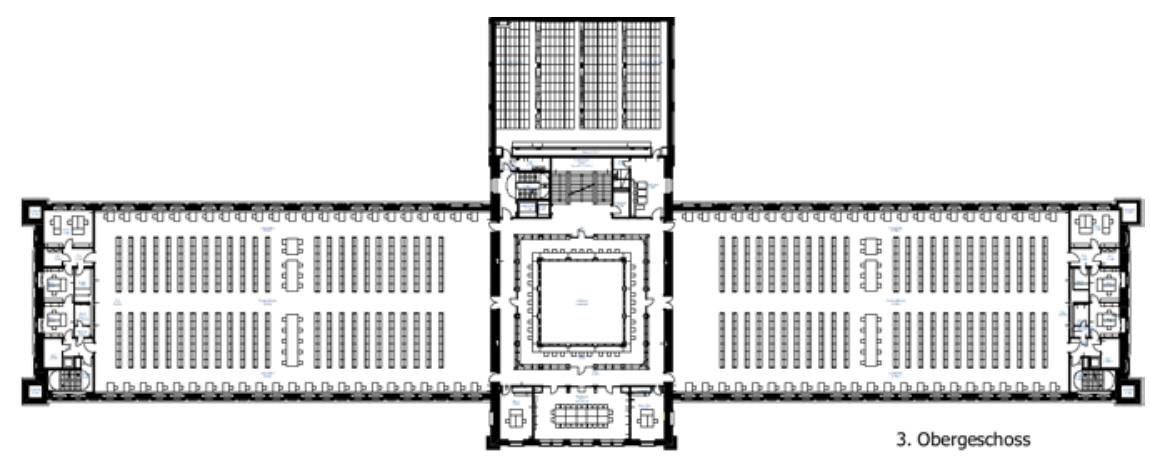

Bild 12 Grundriss des 3. Obergeschosses, Quelle: ARGE Aktienspinnerei 
Im 4. Obergeschoss sind 20 Carrels untergebracht, 16 für je eine Person und 4 für je 2 Personen sowie an der Südseite Büros.

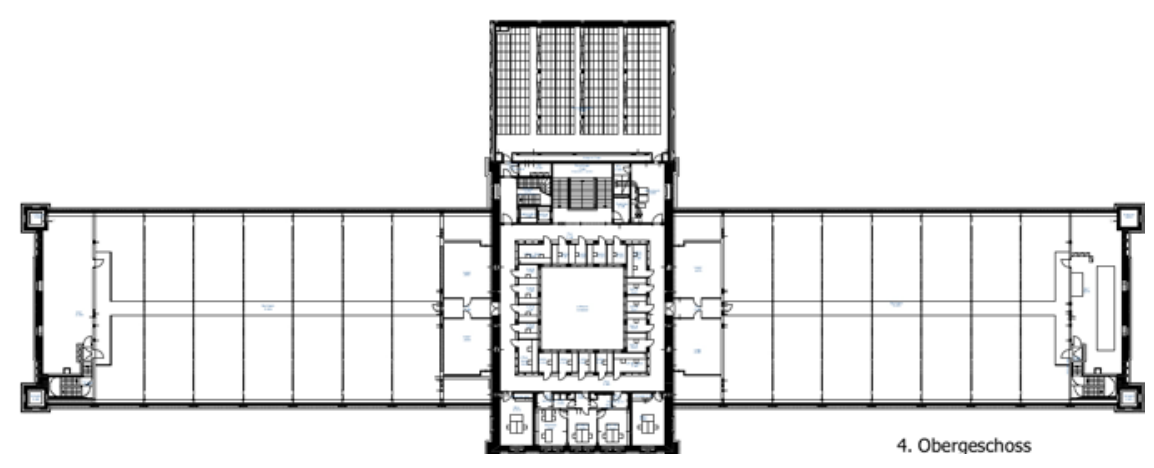

Bild 13 Grundriss des 3. Obergeschosses, Quelle: ARGE Aktienspinnerei

Der Lesesaal erstreckt sich über 3 Etagen, weil sich die Empore des Lesesaales und die Carrels um einen Luftraum schmiegen, der durch eine Glasdecke begrenzt wird.

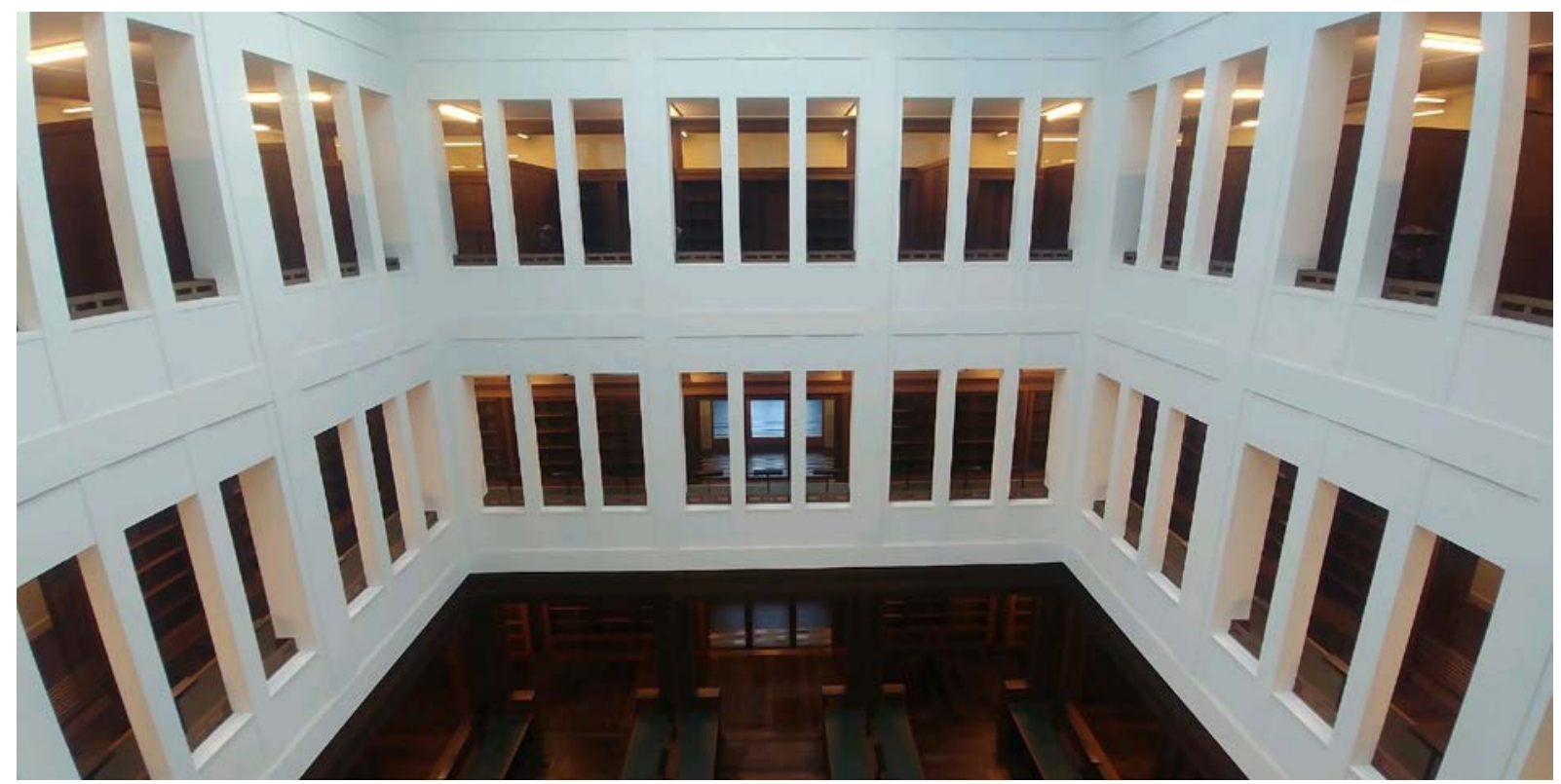

Bild 14 Blick in den Lesesaal, Quelle: TU Chemnitz / A. Malz 


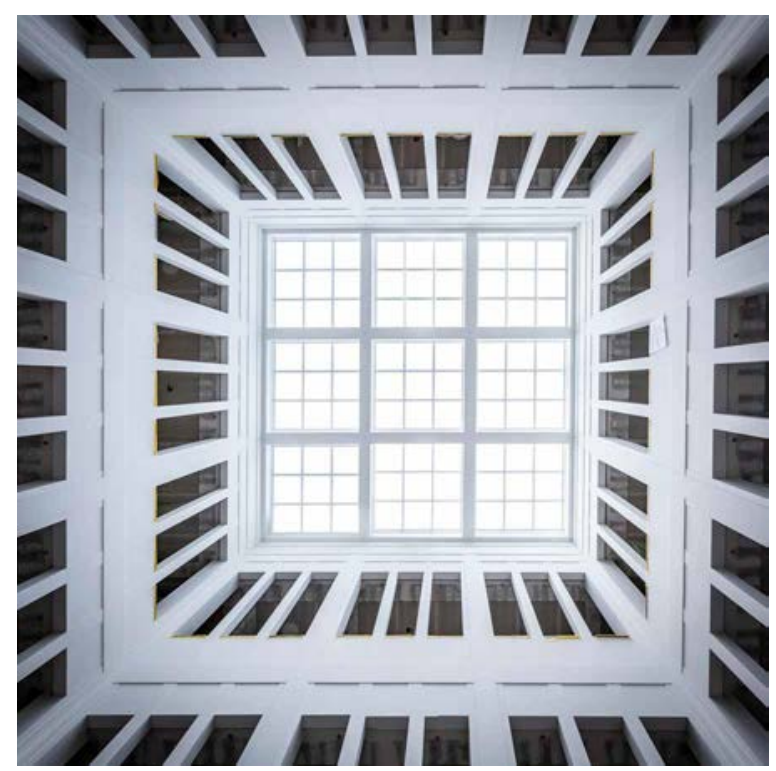

Bild 15 Glasdecke des Lesesaales, Quelle: TU Chemnitz / J. Müller

In allen Geschossen ist an das Mittelteil ein Magazin angeschlossen und vom Erdgeschoss bis zum 3. Obergeschoss sind in den Giebelseiten die Büros für die Mitarbeitenden. Einzelne Büros befinden sich an der Stirnseite des Mittelbaus.

Die Flächen stellen sich wie folgt dar:

2 Seitenflügel je

Mittelbau

Magazin je

Freihand / Lesen

Verwaltung

Magazin

Sonstige

Gesamt
61,50 m x 26,70 m (4 Geschosse ohne Kellergeschoss)

$24,16 \mathrm{~m} \times 38,00 \mathrm{~m}$ (6 Geschosse mit Kellergeschoss)

24,16 m x 18,50 m (6 Geschosse mit Kellergeschoss)

$8520 m^{2}$

$732 \mathrm{~m}^{2}$

$2779 m^{2}$

$323 \mathrm{~m}^{2}$

$12354 \mathrm{~m}^{2}$

Insgesamt können 1450000 Bände untergebracht werden, davon 785000 in der Freihand.

\section{Ausstattung}

Gemäß den Vorgaben des Wettbewerbes zum Umbau der Alten Aktienspinnerei sollte das Haus nicht nur in seiner alten Kubatur wieder aufgebaut werden, sondern es sollte auch der Charakter eines Industriegebäudes gewahrt bleiben.

Dies ist sehr gut gelungen. Alle Details tragen dem industriellen Charakter des Gebäudes Rechnung und sind nah am historischen Vorbild. Dazu gehören die Fenster mit Sprossengliederung sowie die historische Tragkonstruktion der Seitenflügel mit Gusseisenstützen und -trägern, Spanngliedern und Gewölbekappen.

Auch die Materialauswahl entspricht dem Industriecharakter. Für die Hauptfassade wurde feiner Kalkputz verwendet. Die Sockel und Fensterbänke bestehen aus Naturstein (Sandstein). 
Im Inneren wurde für das Foyer grau-beiger Terrazzoboden und für die Freihand- und Bürobereiche Linoleum verwendet. Die Lesebereiche sind mit Massivholzdielen Eiche natur geölt ausgestattet.

Der Kalkputz für die Innenwände ist grauweiß gehalten. Für den Sockel im Foyer und im Windfang des Ostflügels wurde Steinputz verwendet.

Die Holzverkleidung der Wände besteht aus Eiche natur. Für die Ausstattung der Lesebereiche kam Räuchereiche zur Anwendung.

Die Lichtdecke des Lesesaales besteht aus einer Glasdecke mit feiner Metallprofilierung.

Das Farb- und Materialkonzept setzt sich bei der Möblierung fort. Stühle und Tische werden in Eiche natur oder Eiche gebeizt sein. Die Freihandregale sind serienmäßige Stahlsystemregale, ergänzt durch Frontblenden für Stirnseiten und Holzrahmen aus HPL-beschichteten MDF-Platten in schwarzbraun.

Die Planung der Innenausstattung und das Farb- und Materialkonzept hat die ARGE Aktienspinnerei vorgenommen. Seit 2014 gab es regelmäßige Bauberatungen, an denen immer auch Vertreter der Universitätsbibliothek teilnahmen. Auch wenn die Architekten von Anfang an ein sehr klares Bild von der Bibliothek hatten und dieses bis zum Ende durchzogen, waren Veränderungen während der Bauphase möglich. So wurde unter aktiver Einbeziehung der UB-Mitarbeitenden der Thekenbereich umgeplant und es wurden die PC-Arbeitsplätze neu im Gebäude verortet - nämlich als Spange zwischen den Freihandregalen. Der Lern- und Kommunikationsbereich ist am Ende viel flexibler als zu Beginn der Planungen gestaltet.

\section{Technische Ausstattung}

Auch wenn die Hülle des Gebäudes nach altem Vorbild aufgebaut wird, gibt es im Inneren eine moderne technische Ausstattung, die den zeitgemäßen Anforderungen an eine Bibliothek entspricht.

An der Schnittstelle zwischen Altbau und Magazinanbau befindet sich eine Buchtransportanlage, die als Vertikalsystem alle sechs Nutzungsgeschosse der Bibliothek verbindet. Diese Buchtransportanlage, die mit zwei Rückgabeautomaten gekoppelt ist und sechs Mitarbeiterrückgabeplätze haben wird, ist mit 25 Telelift-Fahrzeugen ausgestattet, die jedes Medium einzeln und schonend in die jeweilige Etage befördern. Die angewendete Technologie ist so interessant, dass sich Bibliotheken weltweit dafür interessieren.

Plätze für die Selbstverbuchung der Medien, leistungsfähige Multifunktionsgeräte, moderne PC-Arbeitsplätze, ein leistungsfähiges WLAN und ein intelligentes Türzugangs- und Raumreservierungssystem komplettieren die technische Ausstattung.

Natürlich wird es Aufzüge für die Nutzer geben und zwei Kleingüteraufzüge an den Giebelseiten der Seitenflügel, die die Büros vertikal verbinden. Ein weiterer Kleingüteraufzug befindet sich im Magazinraum des Archives, das mit einem zweigeschossigen Regal ausgestattet ist. 


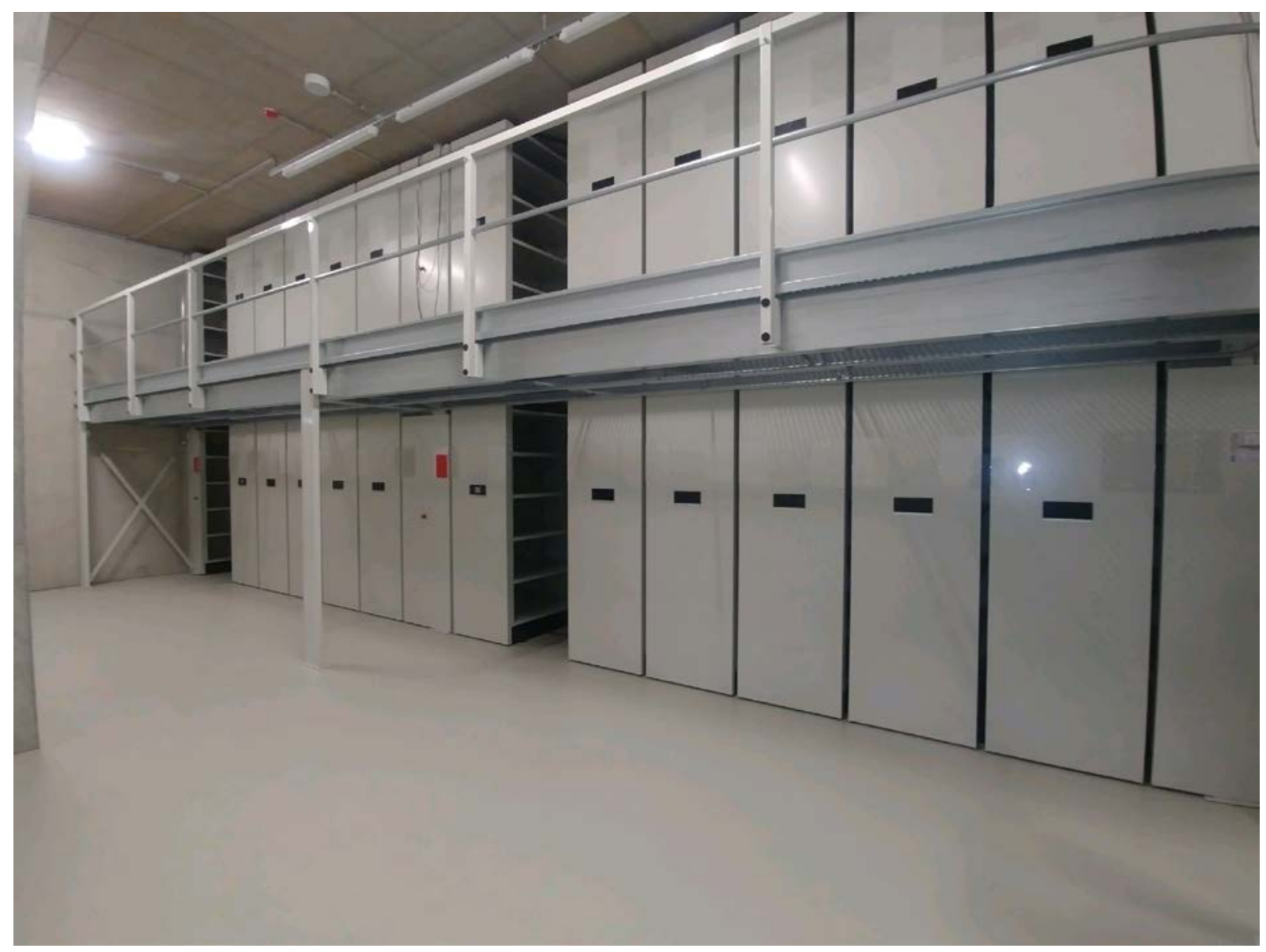

Bild 16 Magazin für das Archiv mit einer zweigeschossigen Regalanlage, Quelle: TU Chemnitz /A. Malz

Die Magazine sind mit elektrisch betriebenen Rollregalen ausgestattet.

Auf den ersten Blick ist nichts sichtbar - trotzdem steckt das Haus voller intelligenter Gebäudetechnik. Einige Beispiele seien hier genannt: ${ }^{1}$

Geheizt wird über eine Fußbodenheizung. Nur in den Büros gibt es Plan-Heizkörper. Die Fläche, die mittels Fußbodenheizung beheizt wird, ist $10200 \mathrm{~m}^{2}$ groß. Das entspricht der Fläche von 2 Fußballfeldern.

Die Kälteversorgung des Gebäudes erfolgt mittels Fernkälte vom Energieversorger eins energie. Es handelt sich um Absorptionskälte, d.h. Kälte mit einer Temperaturspreizung $6 / 12^{\circ} \mathrm{C}$, welche aus überschüssiger Fernwärme gewonnen wird. Ca. 30\% dieser Leistung dienen der direkten Raumkühlung von Seminar- und Gruppenarbeitsräumen sowie technischen Betriebsräumen.

Ca. 70\% der Anschlussleistung werden zur dynamischen Kühlung der Luft in den Raumlufttechnischen-Anlagen (RLT) benötigt.

In den Sommermonaten kann bei anhaltenden Hitzeperioden das für die RLT-Anlagen in den Nachtstunden nicht benötigte Kältepotential (ca. 180 kW Kälteleistung) in die Flächentemperierung der Seitenflügel und des Mittelbaus eingebracht werden.

1 Enderlein,Maik: Kurzbeschreibung der technischen Anlagen der Universitätsbibliothek. Internes Papier des Staatbetriebes Sächsisches Immobilien und Baumanagement, 2020 
Die Umschaltung vom Heiz- in den Kühlbetrieb erfolgt durch die Gebäude-Leittechnik (GLT) über einen zusätzlichen Plattenwärmetauscher zwischen Kälteverteiler und dem Heizkreis der Flächentemperierung nur bei entsprechend langen Hitzeperioden mit Verriegelung der Heiz- und Kühlfunktion.

Für die Klimatisierung kommen 4 Teilklimaanlagen (TKA) mit den Luftbehandlungsfunktionen Filtern, Heizen und Kühlen zum Einsatz. Zwei davon be- und entlüften die Freihandbereiche in den Seitenflügeln. Um die historische Statik mit den gusseisernen Stützen und dem zugstangenunterstützten Kappengewölbe nicht zu stören, wurde auf Kanalinstallation im sichtbaren Bereich komplett verzichtet. Die Zuluft wird an den Stirnseiten über drei großflächige Quellluftauslässe zugfrei in die Etagen eingebracht und verdrängt die vorhandene Raumluft in Richtung der stets offenstehenden Türen zum Mittelbau. Dort wird die Abluft über die im umlaufenden Flur des Mittelbaus befindlichen Quelllufteinlässen abgesaugt und den Zentralgeräten über verdeckt liegende Lüftungskanäle wieder zugeführt.

Die Regelung der Luftleistung erfolgt durch GLT-seitige Auswertung der Luftqualität über je zwei CO2-Sensoren in allen Etagen.

Eine weitere Teilklimaanlage versorgt den Mittelbau (ohne Lesesaal) mit einer Luftleistung von 7900 $\mathrm{m}^{3} / \mathrm{h}$ und eine Teilklimaanlage die Gruppenarbeitsräume im EG Ostflügel mit einer Luftleistung von $6000 \mathrm{~m}^{3} / \mathrm{h}$.

Der Magazinanbau und der Lesesaal werden mit Vollklimaanlagen (VKA) versorgt.

Über die zentrale Gebäudeleittechnik wird das komplexe Zusammenspiel eines Teils der technischen Einzelanlagen wie Klima- und Lüftungsgeräte, Heizungs- und Kälteverteilung sowie die maschinelle Entrauchung geregelt und abgebildet.

Weitere haustechnische Anlagen wie Starkstrom-, Fernmelde- und Informationstechnik, SprinklerSanitär- und Fördertechnik werden in ihrer Funktion auf Störung überwacht.

Einen weiteren Schwerpunkt bildet das Zählermanagement zur Erfassung und Auswertung von Verbräuchen zur energetischen Optimierung des Gebäudes nach Nutzungsbeginn.

Die Stromversorgung erfolgt über Mittelspannungsebene mittels hauseigener Trafostation mit 800 kVA. Es gibt eine Zentralbatterieanlage und unterbrechungsfreie Stromversorgung mit ca. $100 \mathrm{kVA}$ sowie ein Netzersatzaggregat mit 250 kVA.

Eine intelligente, homogene Beleuchtungssteuerung der Lichtdecke im Lesesaal mit automatischem Ausgleich ermöglicht einen künstlichen Anteil in Abhängigkeit zum schwankenden Tageslicht.

Die Allgemeinbeleuchtung erfolgt über eine präsenz- und tageslichtabhängige Steuerung.

Die flächendeckende Brandmeldeanlage (BMA) ermöglicht höchsten Sicherheitsstandard und ist gekoppelt mit einer Sprachalarmierungsanlage. Für 28 Brandszenarien erfolgt eine regelungstechnische Umsetzung. Installiert wurde eine BOS-Funkanlage für die Feuerwehr zur Sicherstellung des Funkempfangs im Gebäude bei einem Löschangriff. Es gibt eine Einbruchmeldeanlage (EMA). Für die Mediensteuerung in den Seminar- und Gruppenräumen wurde ein einheitliches System geschaffen.

Festgelegte Bereiche der Bibliothek werden mittels Videokameras unter Einhaltung der datenschutzrechtlichen Vorschriften überwacht. 
Die Bibliothek wurde als 24/7-Bibliothek geplant, was eine Verdopplung der bisherigen durchschnittlichen Öffnungszeit bedeutet und völlig neue Anforderungen an den Bibliotheksbetrieb stellt.

Deshalb wurde schon in einer sehr frühen Planungsphase mit den Umzugsvorbereitungen begonnen. Diese wurden ohne zusätzliches Personal neben dem Bibliotheksalltag geleistet, was sehr hohe Anforderungen an die UB-Mitarbeitenden stellte. Alle Medien mussten mit RFID-Tags ausgestattet werden, da bisher noch mit Barcodes gearbeitet wurde. Die Bestände wurden sorgfältig analysiert, es gab Aussonderungen und Umsetzungen innerhalb der Bibliotheksstandorte. Denn am Ende des Umzuges sollen Medien aus 3 Standorten in eine Reihenfolge gebracht werden - in der Freihand nach RVK und im Magazin nach numerus currens geordnet. Alle Medien wurden auf Tauglichkeit für die Rückgabe am Rückgabeautomaten und für den Transport über die Buchtransportanlage geprüft und teilweise - vor allem bei Medienpaketen - neu geordnet und beschriftet.

Alle Arbeitsabläufe wurden analysiert und auf die Gegebenheiten in der Alten Aktienspinnerei umgestellt.

Die Aufstellung der Medien in der Freihand und im Magazin in der neuen Bibliothek musste geplant werden. Analysen auf der Grundlage des Bibliothekskataloges aber auch händisches Auszählen waren notwendig, um am Ende einen Plan zu haben, aus dem der Standort jedes einzelnen Mediums im neuen Regal ersichtlich ist.

Das neue Haus bringt neue Ansprüche an die Universitätsbibliothek Chemnitz mit sich. Motor der Stadtentwicklung zu sein, ist nur einer davon.

In der Alten Aktienspinnerei entsteht eine Bibliothek, die mehrere Aufgaben haben wird. Sie ist der Lernort und Treffpunkt der Studierenden und Mitarbeitenden der TU Chemnitz und bietet analoge und digitale Informationen. Sie hält mehr als 700 Plätze für alle Lerngewohnheiten vor. Der klassische Lesesaal lädt zum stillen und konzentrierten Arbeiten ein und im Freihandbereich befinden sich sowohl Leseplätze zum Anlesen als auch Stehplätze. Der Lern- und Kommunikationsbereich lädt zum entspannten Arbeiten ein. Gruppenarbeitsräume mit variablen Möbeln und Carrels für konzentrierte Einzelarbeit runden das Arbeitsplatzangebot ab.

Als öffentliche Bibliothek will sie aber auch der Anlaufpunkt für den an wissenschaftlicher Information interessierten Bürger aus Chemnitz und Umgebung sein. Sie ist gleichzeitig das Informationszentrum für die in Chemnitz und Umgebung ansässigen Unternehmen. Damit wird die UB die Öffnung der Universität nach außen. Durch die Koppelung der Universitätsbibliothek mit dem Universitätsarchiv ist die UB auch die Bewahrerin der Geschichte der TU Chemnitz.

Veranstaltungen sollen stattfinden - gern auch zu Themen, die nicht vordergründig Bibliotheksthemen sein müssen. Ein erstes Event ist schon festgelegt. Am 20.11.2020 wird der Chemnitzer Schmidt-Rottluff-Preis zum zweiten Mal verliehen. Die Arbeiten des Preisträgers werden in der Alten Aktienspinnerei ausgestellt werden.

Zu den bisherigen Serviceangeboten sollen neue kommen. Viele Ideen wurden gesammelt und sind in der Umsetzungsphase. So wird die UB ein neues Logo bekommen, es wird neue buchbare Angebote geben, auch mit Partnern aus der TU Chemnitz. Die Bibliothek möchte „Spielwiese“ für einige an der TU Chemnitz entwickelten Technologien sein.

Nach wie vor will die Bibliothek auf persönliche Angebote setzen. Trotz aller Technik wird es 12 Stunden am Tag UB-Mitarbeitende geben, die den Besucherinnen und Besuchern mit Rat und Tat zur 
Seite stehen werden. In den Nachtstunden und an Sonn- und Feiertagen übernimmt ein Wachdienst die Aufsicht über das Haus.

\section{Chemnitz im Juni 2020}

Aus der grauen, fast schon verfallenen Aktienspinnerei ist ein strahlendes Haus geworden. Die Außenanlagen sind fast fertig und im Inneren des Gebäudes werden Regale und Möbel aufgebaut.

Die ersten Bücher und Zeitschriften sind umgezogen und bis zum Ende des Umzuges werden 33000 m Bibliotheks- und Archivgut, mehrere Hundert PC, Bildschirme, Drucker, Scanner und alle Büromaterialien der Mitarbeiter umgezogen sein.

Am 1.10.2020 soll die Bibliothek eröffnen. Um alle Abläufe sicher zu beherrschen und die Umzüge im Hintergrund des Bibliotheksalltages zu ermöglichen, wird die Zeit bis Ende März 2021 als Probebetrieb definiert. Wenn alles reibungslos funktioniert, wird am 1.4.2021 die UB Chemnitz rund um die Uhr - also 24/7 für ihre Nutzer da sein.

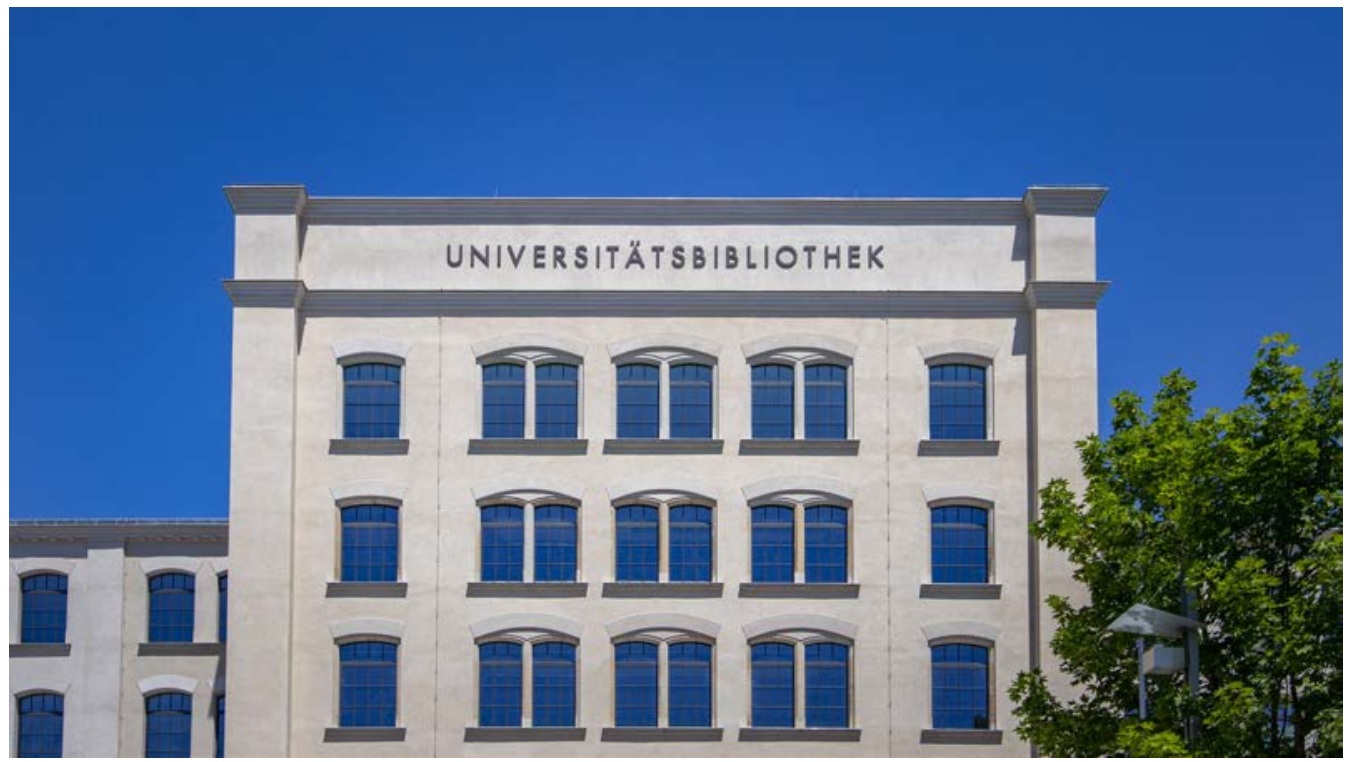

Bild 17 Außenansicht der Alten Aktienspinnerei im Frühjahr 2020,

Quelle: TU Chemnitz / J. Müller 